\title{
Automated Discovery of Compositions of Services Described with Separate Ontologies ${ }^{\star}$
}

\author{
Antonio Brogi ${ }^{1}$, Sara Corfini ${ }^{1}$, José F. Aldana ${ }^{2}$, and Ismael Navas ${ }^{2}$ \\ ${ }^{1}$ Department of Computer Science \\ University of Pisa, Italy \\ ${ }^{2}$ Departamento de Lenguajes y Ciencias de la Computación \\ Universidad de Málaga, Espãna
}

\begin{abstract}
We present a matchmaking system that exploits ontologybased (OWL-S) service descriptions to discover service compositions capable of satisfying a client request. Efficiency is achieved by pre-computing off-line a (hyper)graph that represents the functional dependencies among different (sub)services. The notion of Semantic Field [1] is employed to cross different ontologies.
\end{abstract}

\section{Introduction}

The synergy between Web services and the emerging area of the Semantic Web [2] is promoting the development of so-called semantic Web services. A semantic Web service is a software service which self-describes its functionalities by annotating them with (instances of) concepts defined by means of ontologies.

The development of fully-automated, semantics-based service discovery mechanisms constitutes a major open challenge in this context, and it raises several important issues. One of them is the ability of coping with different ontologies, as different services are typically described in terms of different ontologies. Another important feature is the capability of discovering service compositions rather than single services. Indeed it is often the case that a client query cannot be fulfilled by a single service, while it may be fulfilled by a suitable composition of services. Last, but not least, efficiency is obviously an important objective of service discovery mechanisms.

In this perspective, we proposed in [3] an algorithm for the compositionoriented discovery of (semantic) Web services. The algorithm in [3], as well as its evolved version described in [4, takes a client query, specifying inputs and outputs of the desired service (composition), and performs a flexible matching over a registry of OWL-S advertisements to determine whether there exists a service (composition) capable of satisfying the query. A limitation of 34 is that they do not properly address the problem of crossing ontologies: in 3 all services are (unrealistically) assumed to share the same ontology, while in [4 multiple ontologies are (inefficiently) crossed at query-time.

\footnotetext{
* Work partially supported by the SMEPP project (EU-FP6-IST 0333563) and by the project TIN2005-09098-C05-01 (Spanish Ministry of Education and Science.
} 
In this paper, we extend [3] and [4] by presenting a semantic-based composition-oriented discovery system which employs the notion of Semantic Fields [1 to cross different ontologies, and it achieves efficiency by pre-computing off-line all the query-independent tasks (viz., to determine the dependencies within/among services as well as the relationships among ontologies). The proposed system is the first one - at the best of our knowledge - that addresses all the previously described issues, namely, composition-oriented discovery, ontology crossing and efficiency. The matchmaking system consists of two main modules: the hypergraph builder, which builds a hypergraph representing intraservice and inter-service data relationships, and the query solver, which analyses the hypergraph, given a client query that specifies the set of inputs and outputs of the desired service (composition).

The hypergraph builder and the query solver are described in Sections 2 and 3. respectively. Some concluding remarks are drawn in Section 4 .

\section{Hypergraph Builder}

The hypergraph builder analyses the ontology-based descriptions of the registrypublished services in order to build a labelled directed hypergraph, which synthesises all the data dependencies of the advertised services. Although this module performs a time consuming task, it does not affect the efficiency of the matching process, as the hypergraph construction is completely query independent and can be pre-computed off-line before query answering time.

According to [5, a directed hypergraph $H=(V, E)$ is a pair, where $V$ is a finite set of vertices and $E$ is a set of directed hyperedges. A directed hyperedge is an ordered pair $(X, Y)$ of (possible empty) disjoint subsets of $V$, where $X$ and $Y$ denote the tail and the head of the hyperedge, respectively.

The vertices of the hypergraph constructed by the hypergraph builder correspond to the concepts defined in the ontologies employed by the analysed service descriptions, while the hyperedges represent relationships among such concepts. More precisely, an hyperedge has one of the following three types:

- $E_{\subset}=(D,\{c\}, n i l)-$ subConceptOf relationship. Let $c$ be a concept defined in an ontology $O$ and let $D \in O$ be the set of the (direct) subconcepts of $c$. Then, there is a $E_{\subset}$ hyperedge from $D$ to $c$.

- $E_{\equiv}=(\{e\},\{f\}$, sim $)$ - equivalentConceptOf relationship. Let $e, f$ be two concepts defined in two separate ontologies and let sim be the similarity between $e$ and $f$, i.e., the probability that $e$ is (semantically) equivalent to $f$. As we will see, if $\mathrm{sim}$ is above a given similarity threshold, there is a $E_{\equiv}$ hyperedge from $e$ to $f$ labelled by sim.

- $E_{S}=(I, O,\{s\})$ - intra-service dependency. Let $s$ be (a profile of) a service and let $I$ be the set of inputs that $s$ requires to produce the set $O$ of outputs. Then, there is a $E_{S}$ hyperedge from $I$ to $O$ labelled by $s$.

The hypergraph builder updates the hypergraph whenever a new service $s$ is added to the registry. More precisely, the hypergraph builder firstly adds to the hypergraph the concepts defined in the ontologies employed by $s$ (independently 
of whether they directly occur in the specification of $s$ ). Next, it draws the hyperedges representing the subConceptOf relationships, the equivalentConceptOf relationships, and the intra-service dependencies between the newly added ontology concepts.

As mentioned in the Introduction, in order to cope with different ontologies, the hypergraph builder exploits the notion of Semantic Fields [16], which are groups of interrelated ontologies that may be relevant to a given information request. To find Semantic Fields we firstly compute the similarity between pairs of concepts and next we calculate the distance between pairs of ontologies (i.e., how similar two ontologies are). The Semantic Field Too 1 (SemFiT) determines mappings between concepts by combining the results of individual matchers which analyse the similarity between pairs of concepts with different strategies: exact match, same prefix, same suffix, synonyms in wordNet v.1.7 and similar path in the ontology. The hypergraph builder determines the subConcept $O f$ and equivalentConcept Of relations by suitably exploiting the SemFiT methods.

Consider now the intra-service dependencies of $s$. As one may expect, several intra-service dependencies can be drawn out from a service description, as a service may behave in different ways and feature different functionalities. The different profiles of $s$ can be determined by analysing the OWL- 22 process model which describes the behaviour of $s$. The hypergraph builder inserts in the hypergraph a intra-service dependency for each profile of $s$.

Finally, it is worth observing that the inter-service dependencies are directly represented by the hypergraph and they are automatically updated whenever a service is added to the registry. Indeed, an inter-service dependency between two services $s$ and $t$ occurs if there exists (at least) a concept which belongs both to the head of a $s$-labelled $E_{S}$ hyperedge and to the tail of a $t$-labelled $E_{S}$ hyperedge. Note that there is a inter-service dependency between $s$ and $t$ also if there exist two concepts $c_{s}$ and $c_{t}$ linked together by means of $E_{\subset}$ and/or $E_{\equiv}$ hyperedges, where $c_{s}$ belongs to the head of the $s$-labelled $E_{S}$ hyperedge and $c_{t}$ belongs to the tail of the $t$-labelled $E_{S}$ hyperedge.

We present next an example which illustrates the behaviour of the hypergraph builder. Let us consider an empty registry where we add the hotelService and the conferenceService. The former allows a client to search for and/or to reserve hotels, and the latter allows a client to register to academic events. Figure 1 depicts the OWL-S process models of hotelService and conferenceService, which employ three different ontologies, namely hotel, e-commerce and event 3 .

The hypergraph builder firstly adds to the hypergraph all the concepts defined in the hotel, e-commerce and event ontologies together with the subConcept Of and the equivalentConcept of relationships returned by SemFiT. Then, it computes and inserts also the intra-service dependencies of HotelService and ConferenceService. The resulting hypergraph is shown in Figure 2 .

\footnotetext{
${ }^{1}$ Available through a Web Service at http://khaos.uma.es/SemanticFieldsWS/ services/SemFieldsConceptHierarchy?-wsdl

${ }^{2}$ OWL-S: Semantic Markup for Web Service. http://www.daml.org/services/owl-s

${ }^{3}$ Available at http://khaos.uma.es/esp/ont/br/[hotel, e-commerce, event] .owl
} 


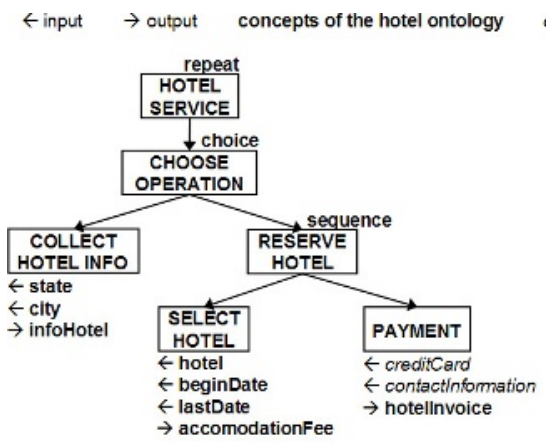

concepts of the e-commerce ontology CONCEPTS OF THE EVENT ONTOLOGY

Fig. 1. Process models of HotelService and ConferenceService

\section{Query Solver}

The query solver takes as input a client query specifying the set of inputs and outputs of the desired service (composition), and next it analyses the hypergraph in order to discover the (compositions of) services capable of satisfying the client request. The formulation of the query is eased by a suitable interface that displays the available concepts (e.g., with an expandable tree structure) and highlights the (computed) equivalences between concepts.

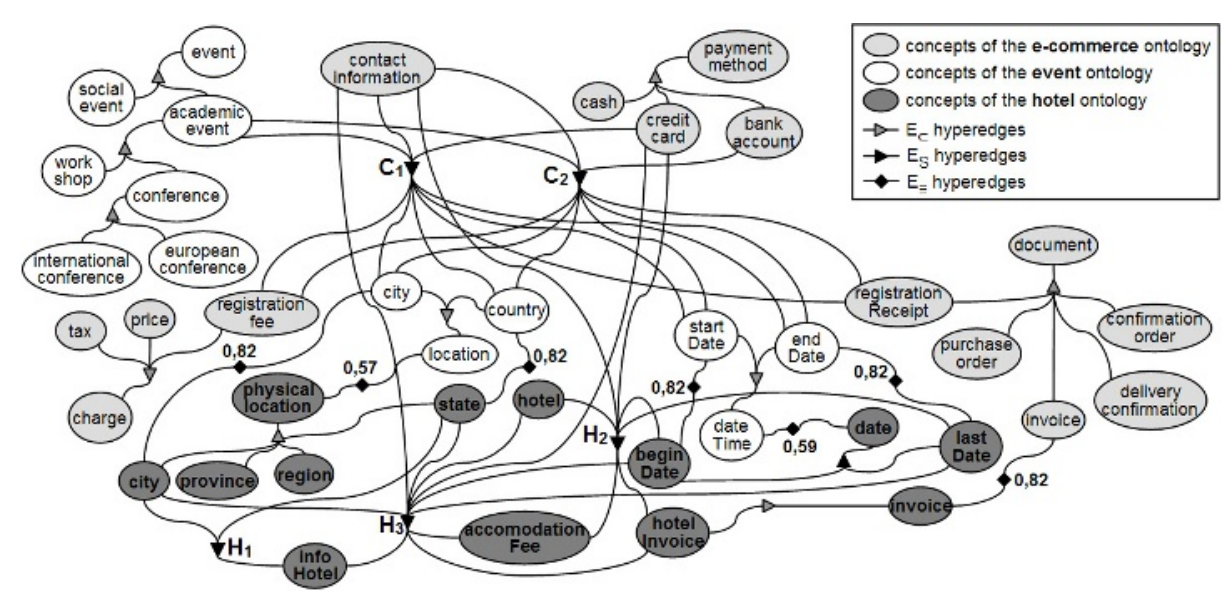

Fig. 2. A simple dependency hypergraph

The query solver explores the hypergraph, by suitably considering the intraservice and inter-service dependencies to address the discovery of (compositions of) services as well as by considering the subConceptOf and equivalentConceptOf relationships to cope with (different) ontologies. Before describing in more details the behaviour of the query solver, let us extend the notion of subConceptOf 
between two concepts $c, d$ defined in two given (possibly equivalent) ontologies. $c$ is subConcept $O f d$ if and only if in the dependency hypergraph there exists a path from $c$ to $d$ which consists of subConcept $O f$ relationships and/or equivalentConcept Of relationships.

The query solver starts by choosing an output $o$ from the goal set (initially the query outputs). A new instance of query solver is generated for each service $s$ which produces (a sub-concept of) $o$. If there exists no service yielding $o$, the query solver fails. Each instance updates the goal set by removing the concepts that are now available (i.e., the outputs of $s$ ) and by adding the inputs of $s$ which are not contained in the query inputs nor produced by some previously selected service. If the goal set is empty, the query solver returns the set of services selected so far (i.e., a successful composition), otherwise it continues recursively. The efficiency of the query solver can be improved by enumerating the non-deterministic generation of the possible solutions in order to return the first successful service (composition) only. Moreover, the large number of services taken into account by the query solver can be reduced by introducing a suitable service pre-selection phase (e.g., using UDDI to filter services not belonging to certain service categories), and by selecting services which produce a needed output with respect to some heuristics (e.g., the number and/or on the quality of the produced outputs) in order to consider the "most promising" services only.

Let us continue the example introduced at the end of Section 2. Consider now a client wishing to plan its participation in an international conference by registering to the conference and by booking her hotel accomodation, and receiving the conference and hotel confirmations. The client query may be composed by the following parameters:

- inputs - event\#internationalConference, hotel\#hotel, e-commerce\#contactInformation, e-commerce\#creditCard

- output - e-commerce\#registrationReceipt, e-commerce\#invoice.

As one may note, neither hotelService nor conferenceService satisfies the given query by itself. Yet, the query can be fulfilled by suitably composing the two available services. Indeed, after visiting the hypergraph in Figure 2, the query solver returns two successful service compositions, represented by the hyperedges $\left\{H_{2}, C_{1}\right\}$ and $\left\{H_{3}, C_{1}\right\}$, while it discards compositions $\left\{H_{2}, C_{2}\right\}$ and $\left\{H_{3}, C_{2}\right\}$, as e-commerce\#bankAccount cannot be produced by any available service.

\section{Concluding Remarks}

We have presented a new fully-automated semantic-based matchmaking system for discovering (compositions of) services capable of satisfying a given client request. As already mentioned in the Introduction, our proposal addresses three main issues of service discovery, namely, composition-oriented discovery, crossing different ontologies and efficiency.

Given the increasing availability of Web services, several composition-oriented discovery systems have been recently proposed, such as 789 , which address the discovery of service compositions by operating in the domain of hypergraphs, 
finite state automata and interface automata, respectively. Although [7/89] deal with ontology-based service descriptions, they do not address the task of crossing ontologies. A composition-oriented discovery algorithm capable of coping with different ontologies has been presented in [10, however, it crosses ontologies at query time, hence severely affecting the efficiency of the whole procedure. An interesting approach for discovering semantic Web services has been proposed in 11, where efficiency is achieved by pre-processing the available ontologies and by pre-classifying the registry-published services before query answering time. Still, 11 does not address the discovery of service compositions.

Our plan for future work includes: to develop fresh indexing and/or ranking techniques to sensibly improve the efficiency of the query solver, to complete our system by employing a behavioural analyser module to determine whether the candidate services can really be composed together and satisfy the query without dead-locking, and finally, to extend SemFiT by employing other existing matching tools in order to achieve better results for the ontology matching problem. Our long-term goal is to develop a well-founded methodology to support an efficient and fully-automated discovery and composition of Web services.

\section{References}

1. Navas-Delgado, I., Sanz, I., Aldana-Montes, J.F., Berlanga, R.: Automatic Generation of Semantic Fields for Resource Discovery in the Semantic Web. In: 16th Int. Conf. on Database and Expert Systems Applications. LNCS 3588. (2005)

2. Berners-Lee, T., Hendler, J., Lassila, O.: The Semantic Web. In: Scientific American. (2001)

3. Brogi, A., Corfini, S., Popescu, R.: Composition-oriented Service Discovery. In Gschwind, T., Aßmann, U., Nierstrasz, O., eds.: Software Composition. LNCS 3628, Springer-Verlag (2005) 15-30

4. Brogi, A., Corfini, S.: Behaviour-aware discovery of Web service compositions. In: University of Pisa, Department of Computer Science - Tech. Rep. TR-06-08. (2006)

5. Gallo, G., Longo, G., Nguyen, S., Pallottino, S.: Directed hypergraphs and applications. Discrete Applied Mathematics 42 (1993) 177-201

6. Aldana-Montes, J.F., Navas-Delgado, I., del Mar Roldan-Garcia, M.: Solving Queries over Semantically Integrated Biological Data Sources. In: Int. Conf. on Web-Age Information Management (WAIM 2004). LNCS 3129. (2004)

7. Benatallah, B., Hacid, M.S., Rey, C., Toumani, F.: Request Rewriting-Based Web Service Discovery. In Goos, G., Hartmanis, J., van Leeuwen, J., eds.: The Semantic Web - ISWC 2003, LNCS 2870, Springer-Verlag (2003) 242-257

8. Mokhtar, S.B., Georgantas, N., Issarny, V.: Ad Hoc Composition of User Tasks in Pervasive Computing Environment. In Gschwind, T., Aßmann, U., Nierstrasz, O., eds.: Software Composition, LNCS 3628, Springer-Verlag (2005)

9. Hashemian, S., Mavaddat, F.: A Graph-Based Approach to Web Services Composition. In IEEE Computer Society, ed.: SAINT 2005, CS Press (2005) 183-189

10. Aversano, L., Canfora, G., Ciampi, A.: An Algorithm for Web Service Discovery through Their Composition. In Zhang, L., ed.: IEEE International Conference on Web Services (ICWS'04), IEEE Computer Society (2004) 332-341

11. Mokhtar, S.B., Kaul, A., Georgantas, N., Issarny, V.: Towards Efficient Matching of Semantic Web Service Capabilities. In: Proceedings of WS-MATE 2006. (2006) 